\title{
Guía colombiana para el diagnóstico de la deficiencia de lipasa ácida
}

\section{Colombian Guidelines for Diagnosis of Lipase Acid Deficiency}

\author{
Alfredo Santamaría Escobar, ${ }^{1}$ Carolina Baquero Montoya, ${ }^{2}$ Roberto García Bermejo, ${ }^{3}$ Adriana María Jaramillo Villegas, ${ }^{4}$ Armando Barrios, ${ }^{5}$ \\ Mauricio Coll Barrios, ${ }^{6}$ Rodrigo de Vivero Camacho, ${ }^{7}$ Rocío del Pilar López Panqueva, ${ }^{8}$ William Márquez, ${ }^{9}$ Diana Victoria Mora Quintero, ${ }^{10}$ \\ Omar Pérez Álvarez, ${ }^{11}$ Carolina Rivera Nieto, ${ }^{12}$ Evelyn Simancas Escalante, ${ }^{13}$ Giomar Sarmiento Marengo, ${ }^{14}$ Luis Carlos Ramírez Urrego, ${ }^{15}$ \\ Patricia Ruiz Navas, ${ }^{16}$ Claudia Sánchez Franco ${ }_{17}^{17}$ David Alfonso Suárez Zamora, ${ }^{18}$ Verónica Botero. ${ }^{19}$
}

Hospital Universitario San Vicente Fundación, Medellín, Colombia.

2 Hospital Pablo Tobón Uribe, Medellín, Colombia.

3 Hospital Infantil Napoleón Franco Pareja, Universidad del Sinú, Cartagena, Colombia.

${ }^{4}$ Clínica Las Américas, Medellín, Colombia.

5 Unidad de Gastroenterología Pediátrica del Cesar, Valledupar, Colombia.

${ }^{6}$ Centro Médico Almirante Colón, Bogotá, Colombia.

7 Hospital Infantil Napoleón Franco Pareja. Departamento de Pediatría, Universidad de Cartagena, Cartagena, Colombia.

8 Hospital Universitario Fundación Santa Fe de Bogotá Universidad de los Andes, Bogotá, Colombia.

9 Fundación Hospital de la Misericordia, Bogotá, Colombia.

${ }^{10}$ Fundación Hospital de la Misericordia, Bogotá, Colombia.

${ }^{11}$ Clínica la Misericordia, Barranquilla, Colombia

${ }^{12}$ Fundación Cardioinfantil, Bogotá, Colombia.

Correspondencia: carolinariveran@gmail.com.

${ }^{13}$ Hospital San Juan de Sahagún. Sahagún, Córdoba, Colombia.

${ }^{14}$ Hospital Universitario Fernando Troconis, Santa Marta, Magdalena, Colombia.

${ }^{15}$ Unidad Maternoinfantil del Tolima, Hospital Federico

Lleras Acosta, Ibagué, Tolima, Colombia.

${ }^{16}$ Hospital Pablo Tobón Uribe, Medellín, Colombia.

${ }^{17}$ Fundación Hospital de la Misericordia, Bogotá

Colombia.

${ }^{18}$ Hospital Universitario Fundación Santa Fe de Bogotá, Bogotá, Colombia.

${ }^{19}$ Fundación Valle del Lili, Cali, Colombia.

\section{Resumen}

Introducción: la deficiencia de lipasa ácida lisosomal (LAL-D) es una entidad de herencia autosómica recesiva que lleva a la acumulación de esteres de colesterol y triglicéridos en el hígado, bazo y otros órganos. La edad de inicio y la tasa de progresión son muy variables, lo que posiblemente sea explicado por las mutaciones presentes en el gen LIPA. Las manifestaciones clínicas son las mismas que para otras patologías hepáticas, cardiovasculares y metabólicas, lo que hace difícil reconocerla en la práctica clínica. Objetivo: proveer una guía que permita a los clínicos reconocer los principales grupos de riesgo en los cuales se debe sospechar de LAL-D y mejorar su diagnóstico. Metodología: este documento se diseñó como un consenso de expertos en el cual participaron médicos especialistas en gastroenterología, hepatología, endocrinología, genética, patología y pediatría. Se realizó una revisión de la literatura acerca de las manifestaciones clínicas y de las herramientas para el diagnóstico de LAL-D y se siguió la metodología de técnica de grupo nominal. Resultados: se generaron algoritmos diagnósticos por consenso para cada uno de los grupos de riesgo, que facilitaran la sospecha y el diagnóstico de LAL-D. Conclusiones: esta guía propone algoritmos para el diagnóstico de LAL-D con base en el consenso clínico, que buscan optimizar la ruta diagnóstica en los pacientes con dicha patología.

\section{Palabras clave}

Deficiencia de lipasa ácida, enfermedad por depósito de esteres de colesterol, enfermedad de Wolman, dislipidemia, hepatomegalia.

\begin{abstract}
Introduction: Lysosomal acid lipase deficiency (LAL-D) is an inherited autosomal recessive entity that leads to the accumulation of cholesterol and triglyceride esters in the liver, spleen and other organs. The age of onset and rate of progression vary greatly, possibly explained by mutations of the LIPA gene. Clinical manifestations are the same as those of other hepatic, cardiovascular and metabolic pathologies which makes it difficult to recognize in clinical practice. Objective: The objectives of these guidelines is to help clinicians recognize the major groups at risk for LAL-D and to improve its diagnosis. Methodology: This document was designed as a consensus of experts in gastroenterology, hepatology, endocrinology, genetics, pathology and pediatrics. A review of the literature regarding clinical manifestations and tools for diagnosis of LAL-D was conducted and the nominal group technique was followed. Results: Diagnostic algorithms which facilitate suspicion and diagnosis of LAL-D were generated by consensus for each of the risk groups. Conclusions: This guide proposes algorithms for the diagnosis of LAL-D based on clinical consensus. The algorithms seek to optimize diagnosis for patients with this pathology.
\end{abstract}

\section{Keywords}

Acid lipase deficiency, cholesterol ester deposition disease, Wolman's disease, dyslipidemia, hepatomegaly. 


\section{INTRODUCCIÓN}

Las guías y los consensos de expertos son documentos que tienen como objetivo ayudar a los médicos a evaluar los beneficios y los riesgos de procedimientos diagnósticos y terapéuticos relacionados con una patología específica. Estos documentos no pretenden reemplazar el criterio y la autonomía médicos, sino ser útiles para la toma de decisiones clínicas. La presente guía fue el resultado de un consenso de 20 expertos de diferentes especialidades médicas.

Cada uno de los grupos de especialistas realizó una búsqueda exhaustiva de la evidencia disponible en la literatura médica actual, mediante los buscadores más utilizados (PubMed y LILACS) y se llevó a cabo una revisión y síntesis de la evidencia, que fue la base para el consenso de expertos. Cada grupo elaboró un documento con el tema afín y se envió, vía correo electrónico, a todos los expertos para su conocimiento, análisis y aportes. En una reunión presencial los expertos presentaron, mediante una conferencia científica, la mejor evidencia disponible sobre los criterios diagnósticos para LAL-D en cada grupo de riesgo; luego, se realizó un debate en el que cada participante pudo expresar su opinión. Una vez analizados los puntos de debate, se llegó a un acuerdo consensuado de más del $80 \%$ de los miembros para, posteriormente, emitir un informe con las recomendaciones y el diseño de algoritmos que definieron la ruta para el diagnóstico de esta patología en los diferentes grupos de riesgo, que estuvo a cargo de los coordinadores del consenso. Este documento luego se elevó para el consentimiento de todos los participantes.

La LAL-D (OMIM 278000) es una enfermedad autosómica recesiva causada por mutaciones en el gen LIPA que reducen drásticamente la actividad de la enzima lipasa ácida lisosomal (LAL; EC 3.1.1.13) (1). Esta enzima es la encargada de clivar los esteres de colesterol y los triglicéridos; por tanto, su disfunción lleva a la acumulación de estos en el hígado, bazo, macrófagos y otros órganos (2). La enfermedad se caracteriza por una esteatosis microvesicular que produce insuficiencia hepática, elevación de las transaminasas séricas, ateroesclerosis acelerada y muerte prematura (3). La presentación infantil se describió en 1956 y recibió históricamente el nombre de enfermedad de Wolman (4). Se caracteriza por vómito, diarrea, falla del crecimiento y hepatoesplenomegalia, con un rápido deterioro de la función hepática y fallecimiento en promedio a los 3,7 meses (5). La forma de inicio tardío se conocía como enfermedad por depósito de esteres de colesterilo y se describió en 1963 (6). Esta tiene un espectro clínico amplio y puede presentarse en bebés, niños o adultos (7). Usualmente es subdiagnosticada y cursa con compromiso hepático, transaminasas elevadas y niveles séricos elevados de colesterol LDL (lipoproteína de baja densidad) y triglicéridos, con concentraciones normales o bajas de colesterol HDL (lipoproteína de alta densidad). El fallecimiento precoz se debe a la insuficiencia hepática o a la ateroesclerosis acelerada secundaria a la hiperlipidemia crónica (2).

La incidencia se ha estimado, a partir de estudios genéticos, en 1/40 $000(8,9)$, mientras que las formas más severas tienen una incidencia aproximada de 1/500 000 (10). Dada la presentación clínica inespecífica y el relativo bajo número de casos publicados, se cree que esta entidad tiene un alto índice de subdiagnóstico (11). Es por esto que los desarrolladores de esta guía pretenden ofrecer al personal del área de la salud una serie de recomendaciones que les permitan guiarse en la sospecha y el diagnóstico de los pacientes con LAL-D.

Esta guía se desarrolló con una técnica de grupo nominal mediante un comité de expertos de diferentes especialidades (pediatría, gastroenterología pediátrica, endocrinología pediátrica, hepatología, patología y genética) quienes, basados en su experiencia y en una amplia revisión de la literatura, expusieron sus diferentes puntos de vista para establecer un listado de recomendaciones mediante debate y discusión, facilitando el juicio colectivo, cuyo objetivo fue proponer una guía que permita la identificación de pacientes con esta patología en Colombia y que pueda usarse en la práctica clínica.

\section{FISIOPATOLOGÍA}

La LAL-D se origina por mutaciones en el gen LIPA, localizado en 10q23.2, tiene 10 exones y una longitud aproximada de 45 kilobases (kb) (1). Esta entidad es de herencia autosómica recesiva, el defecto más común está asociado con una mutación en el sitio del exón 8 (c.894G>A), conocida como E8SJM, que está presente en cerca de la mitad de los pacientes afectados (12). Hasta la fecha se han descrito más de 40 mutaciones relacionadas con la patología (2); estas variantes llevan a la alteración en la función de la enzima. Las más drásticas (nonsense y frameshift) usualmente se asocian con pacientes severamente afectados, mientras que los casos en niños y adultos se relacionan con mutaciones cuyo efecto en la proteína no es tan dramático (13).

La enzima LAL desempeña un papel clave en el metabolismo de los lípidos, ya que es la encargada de la hidrólisis de los esteres de colesterol y los triglicéridos a nivel lisosomal (14). La acción de esta enzima genera colesterol libre, que está involucrado en la regulación negativa de los receptores de LDL, en la inhibición de la enzima 3-hidroxi3-metilglutaril-coenzima A reductasa y en la estimulación de la acil-coenzima A-colesterol-aciltransferasa. La deficiencia congénita de esta enzima lleva a una acumulación lisosomal de esteres de colesterol y triglicéridos, así como a una falla en la vía del receptor LDL. Por tanto, además de esta acumula- 
ción lisosomal masiva de esteres de colesterol, se produce un aumento sistémico de la síntesis de colesterol (15).

\section{CUADRO CLÍNICO}

La deficiencia de lipasa ácida tiene un amplio rango de manifestaciones fenotípicas, que pueden ser la presentación infantil temprana, una aparición durante la infancia o en etapas tardías de la vida.

\section{Presentación infantil}

Usualmente cursa con síntomas más agudos que pueden manifestarse en los primeros días de vida. Los síntomas gastrointestinales incluyen vómito, diarrea, esteatorrea y distensión abdominal secundaria a la hepatoesplenomegalia, así como falla en el crecimiento (10). El aumento en los depósitos de lípidos en el tracto gastrointestinal produce un engrosamiento del intestino resultando en malnutrición y un síndrome de mala absorción (16). La calcificación de las glándulas suprarrenales es característica de esta patología, puede estar presente hasta en el 78\% de los niños afectados y causar insuficiencia adrenal cortical (17).

Los pacientes progresan a una falla multiorgánica, particularmente con falla hepática, cirrosis y caquexia, lo que genera una muerte temprana: en promedio, a los 3,7 meses (5).

\section{Presentación en niños y adultos}

Esta puede cursar de manera similar a la infantil. Un tercio de los pacientes experimentan síntomas gastrointestinales severos, incluyendo diarrea frecuente, vómito, dolor abdominal, malabsorción y esteatorrea $(2,14)$, así como colestasis, falla en el crecimiento, disfunción de la vesícula biliar, entre otros (2). Clásicamente, cursan con dislipidemia, aumento del colesterol total, elevación de LDL y de apolipoproteína $\mathrm{B}$, así como disminución de los niveles de $\operatorname{HDL}(2,14)$. Esta hiperlipidemia se asocia con ateroesclerosis, enfermedad arterial coronaria y eventos vasculares catastróficos tempranos $(2,18)$. En ocasiones pueden ser visibles los depósitos de colesterol (p. ej., xantelasma, particularmente en la región palpebral) (19).

La hepatomegalia con o sin esplenomegalia es frecuente y está presente en el $99 \%$ de los niños y $74 \%$ de los adultos con LAL-D (2). Usualmente, la organomegalia puede ser el primer hallazgo y está presente muchos años antes de que se realice el diagnóstico (20). Los pacientes con esplenomegalia pueden desarrollar anemia o trombocitopenia secundarias al hiperesplenismo.

El compromiso hepático es común y los niveles elevados de alanina aminotransferasa (ALT) y aspartato aminotransferasa (AST) pueden ser el indicio temprano del daño hepático. Este puede manifestarse con o sin ictericia, esteatosis, fibrosis o cirrosis. El daño hepático puede llevar a várices esofágicas y progresar a falla hepática, cirrosis o carcinoma hepatocelular (21).

Los depósitos de lípidos a nivel intestinal pueden llevar a diarrea y pérdida de peso (22).

\section{DIAGNÓSTICO}

El diagnóstico de LAL-D puede realizarse a través de la medición de la actividad de la enzima lipasa ácida o por la determinación de mutaciones en el gen LIPA (14).

- Medición de la actividad de LAL: esta se realiza con una gota de sangre seca (DBS) (23) usando como sustrato fluorimétrico 4-metil lumbiferil palmitato. Debido a que la presencia de otras lipasas en la sangre puede interferir con la medición, se utiliza un inhibidor de LAL, Lalistat 2 (Chemical Tools, South Bend, IN, Estados Unidos) (23). La actividad de LAL se determina comparando la actividad de la lipasa total con la actividad de lipasa en presencia del inhibidor; la diferencia entre las 2 es la atribuible a la enzima LAL. Esta medición puede realizarse en leucocitos y en DBS, ambas cuentan con valores similares de sensibilidad y especificidad (24). Sin embargo, la medición en DBS ha demostrado una excelente discriminación entre sanos y afectados, con valores intermedios para los portadores y cuenta con la ventaja de ser fácil de tomar y transportar, y es muy estable a largo plazo $(23,24)$.

- Estudio molecular: la secuenciación completa de la región codificante del gen LIPA permite la identificación de las mutaciones causales de la enfermedad. La mayoría de los pacientes son homocigotos o heterocigotos compuestos. Sin embargo, algunos pacientes pueden tener mutaciones intrónicas que pueden no ser detectadas en la secuenciación (14). Por tanto, el método de elección para el diagnóstico es la medición de la actividad enzimática y las pruebas moleculares se consideran complementarias.

La Figura 1 resume el algoritmo para el diagnóstico de LAL-D.

Otras pruebas pueden ser utilizadas en la sospecha de la patología, pero no confirman el diagnóstico.

- Biopsia hepática: típicamente, los pacientes van a cursar con esteatosis microvesicular o mixta; sin embargo, no es exclusivo de LAL-D. Otros hallazgos como la presencia de histiocitos en "mar azul", aumento en el tamaño de las células de Kupffer con aumento de vacuolas, gotas de lípido y cristales de colesterol pueden soportar el diagnóstico (9). A nivel de inmunohistoquímica se pueden usar marcadores lisosomales de catepsina $\mathrm{D}$ y 


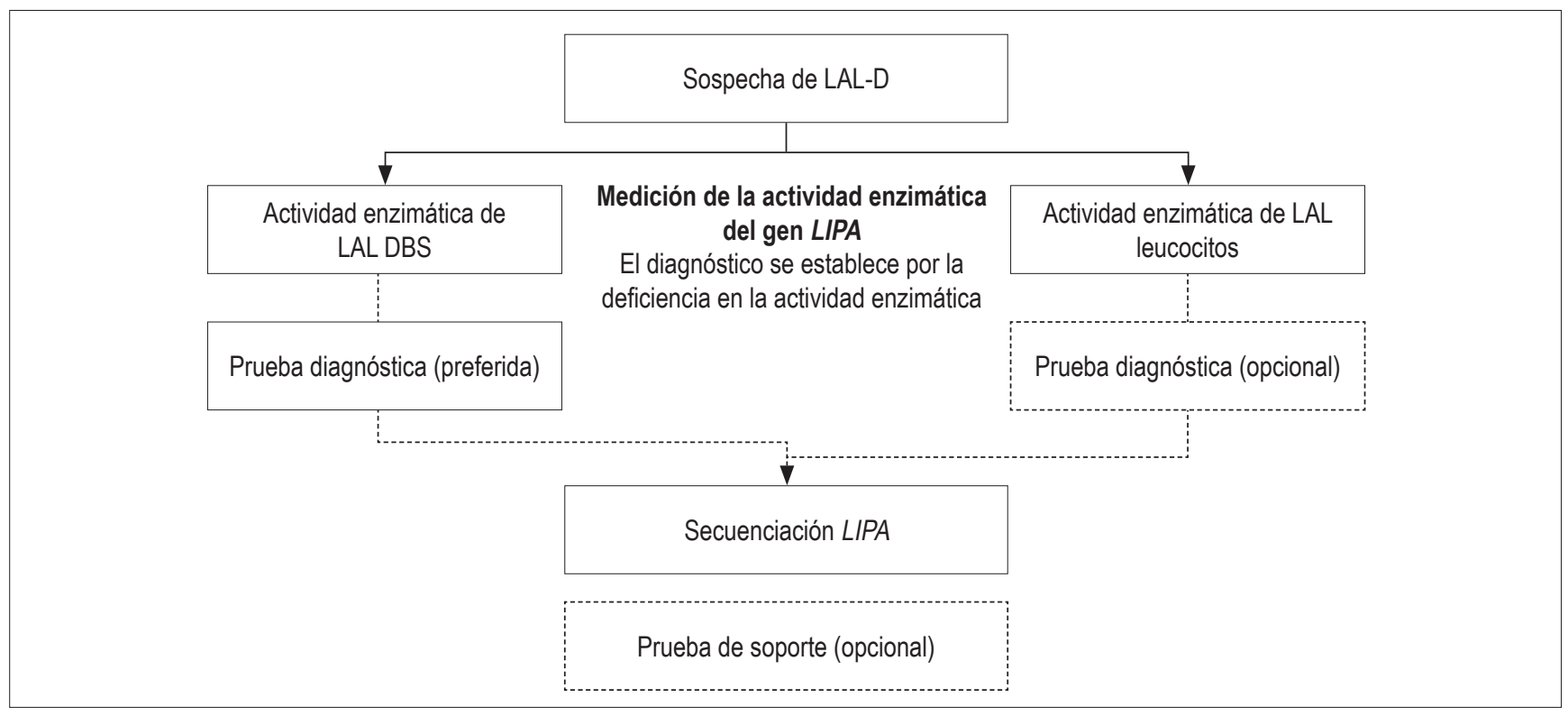

Figura 1. Algoritmo para el diagnóstico de LAL-D.

proteínas 1 y 2 asociadas con la membrana lisosomal (LAMP1 y LAMP2) (25).

\section{DEFINICIÓN DE GRUPOS DE RIESGO}

Debido a que LAL-D cursa con un cuadro clínico similar con otras enfermedades cardiovasculares, hepáticas y metabólicas, es posible que se llegue a diagnósticos inapropiados y a un retraso en el manejo de estos pacientes.

Teniendo esto en cuenta, se decidió establecer para cada uno de los grupos de riesgo un algoritmo que permitiera establecer en qué momento del proceso diagnóstico debía realizarse la prueba de la actividad de la enzima LAL. A continuación se presenta el marco teórico y las rutas diagnósticas definidas.

\section{Enfermedades hepáticas}

\section{Enfermedad por hígado graso no alcohólico}

Esta entidad requiere de la evidencia de esteatosis hepática, por imágenes o histología, y de que no exista una causa para la acumulación de grasa hepática como el consumo de alcohol, el uso de medicación esteatogénica o trastornos hereditarios. En la mayoría de los casos se asocia con factores de riesgo metabólico como obesidad, diabetes mellitus (DM) y dislipidemia. Esta a su vez se subdivide en hígado graso no alcohólico (NAFLD) y esteatohepatitis no alcohólica (NASH). El NAFLD se define como la presencia de esteatosis hepática sin evidencia de daño hepatocelular. La NASH se describe como la presencia de esteatosis hepática e inflamación, daño en el hepatocito con o sin fibrosis (26).

Las causas comunes de esteatosis hepática incluyen un consumo significativo de alcohol, hepatitis $\mathrm{C}$, fibrosis quística, medicación, nutrición parenteral, hepatitis autoinmune, medicamentos (etanol, corticoides, estrógenos, entre otros) y malnutrición severa, entre otras. Estas deben descartarse en el estudio inicial del paciente realizando un perfil viral/inmunológico completo para excluir los trastornos más comunes.

Se debe sospechar LAL-D en pacientes con NASH o NAFLD que cursen con:

- Persistencia en transaminasas elevadas a pesar de pérdida de peso.

- Dislipidemia: aumento en los valores de LDL y disminución de HDL según la edad (que no mejoran luego del cambio en el estilo de vida o la medicación).

- Rápida progresión de la enfermedad.

La Figura 2 muestra el algoritmo para el diagnóstico de LAL-D en pacientes con hígado graso.

\section{Hepatomegalia}

El tamaño hepático normal se basa en valores establecidos mediante percusión, de extensión por debajo del reborde costal derecho o la longitud del eje vertical estimado por estudios de imagen. En general, se estima que los valores 


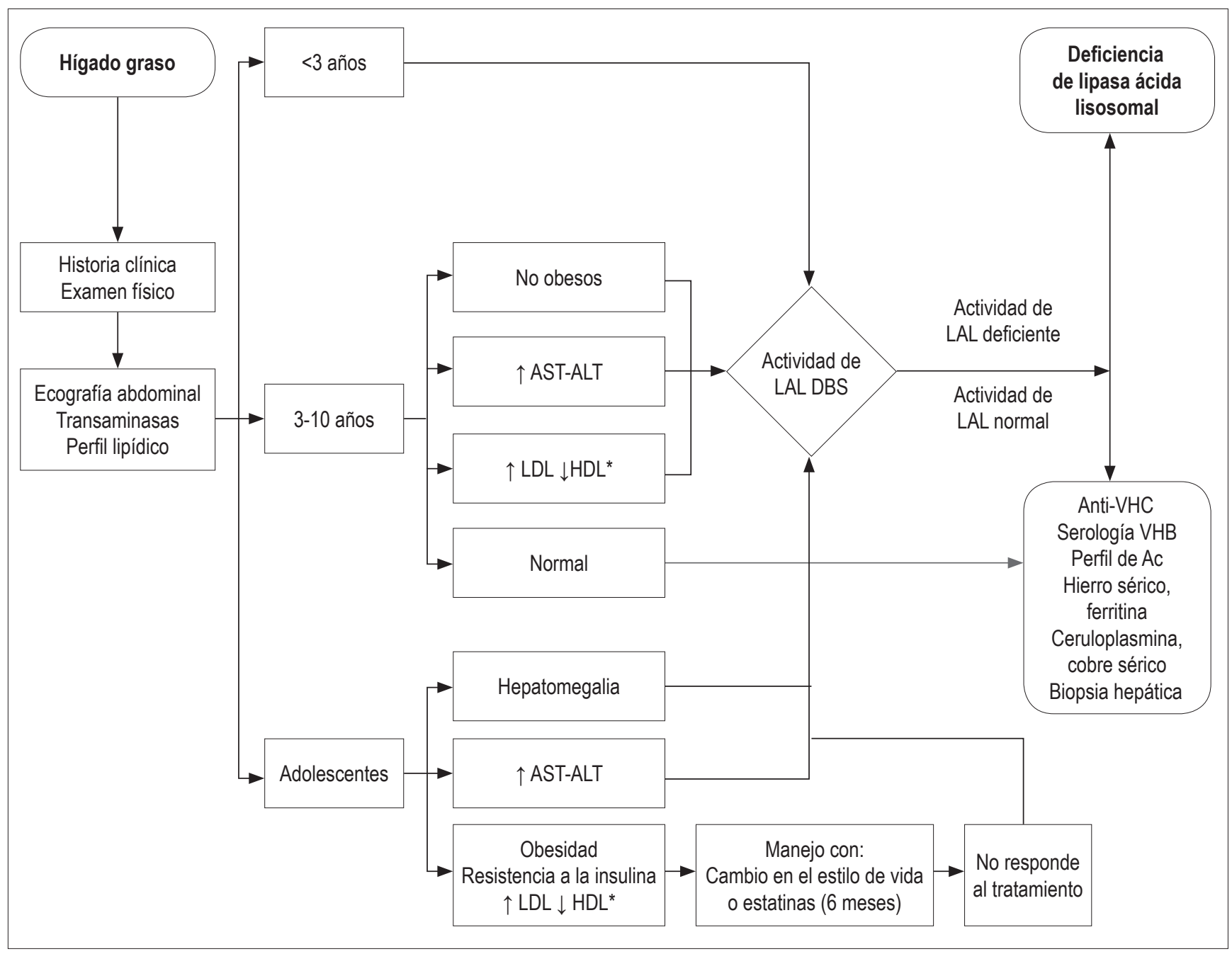

Figura 2. Algoritmo para la sospecha de LAL-D ante la presencia de hígado graso. ${ }^{*} \mathrm{LDL}(\mathrm{mg} / \mathrm{dL}) \geq 130$ o HDL (mg/dL) $\leq 45$. Ac: anticuerpos; AntiVHC: anticuerpos anti-virus de la hepatitis C; VHB: virus de la hepatitis B.

superiores a 3,5 cm en neonatos y a $2 \mathrm{~cm}$ en niños, obtenidos mediante la palpación realizada por debajo del reborde costal derecho en la línea media clavicular, son indicativos de hepatomegalia (27).

En cuanto a su etiopatogenia, la hepatomegalia puede ser originada por 5 mecanismos diferentes: inflamación, depósito excesivo, infiltración, congestión y obstrucción.

1. Inflamación: usualmente secundaria a infecciones, los tóxicos, las radiaciones, enfermedades autoinmunes y la hiperplasia de células de Kupffer que llevan a una hepatomegalia de tipo inflamatorio.

2. Depósito: principalmente de glucógeno, lípidos, grasa, metales y proteínas anormales.

3. Infiltración: es el caso de tumores, quistes parasitarios y hematopoyesis extramedular.
4. Congestión vascular: por obstrucción al drenaje venoso entre el hígado y la aurícula derecha, puede localizarse a nivel intrahepático o extrahepático.

5. Obstrucción biliar: se da en atresia biliar, quistes de colédoco, colelitiasis y tumores de hígado, vía biliar, páncreas y duodeno (27).

La anamnesis y la exploración física, junto con las pruebas complementarias básicas (hemograma, coagulación, función hepática, gasometría, sedimento urinario y ecografía), permiten orientar al paciente pediátrico con hepatomegalia y seleccionar las demás pruebas complementarias a realizar.

A continuación se proponen los algoritmos diagnósticos para las transaminasas (Figura 3) y la hepatomegalia (Figura 4). 


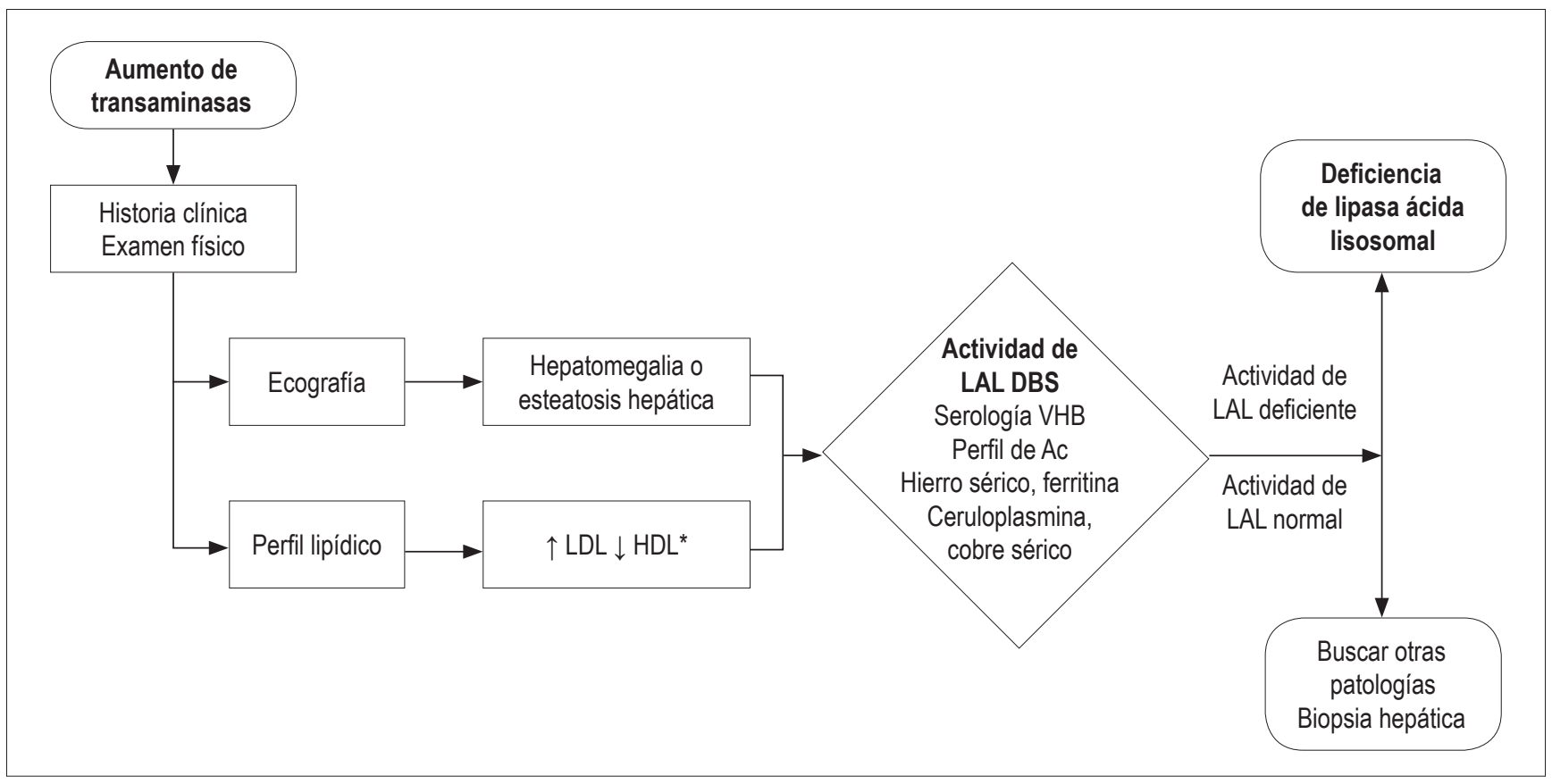

Figura 3. Algoritmo para la sospecha de LAL-D ante la presencia de transaminasas elevadas. *Pediátrica: LDL (mg/dL) $\geq 130 \mathrm{o} \mathrm{HDL}(\mathrm{mg} / \mathrm{dL}) \leq 45$; adulta: $\mathrm{LDL}(\mathrm{mg} / \mathrm{dL}) \geq 160 \mathrm{o} \mathrm{HDL}(\mathrm{mg} / \mathrm{dL}) \leq 40$ (hombres)/ $\leq 50$ (mujeres). Ac: anticuerpos; Anti-VHC: anticuerpos antivirus de la hepatitis C; VHB: virus de la hepatitis B.

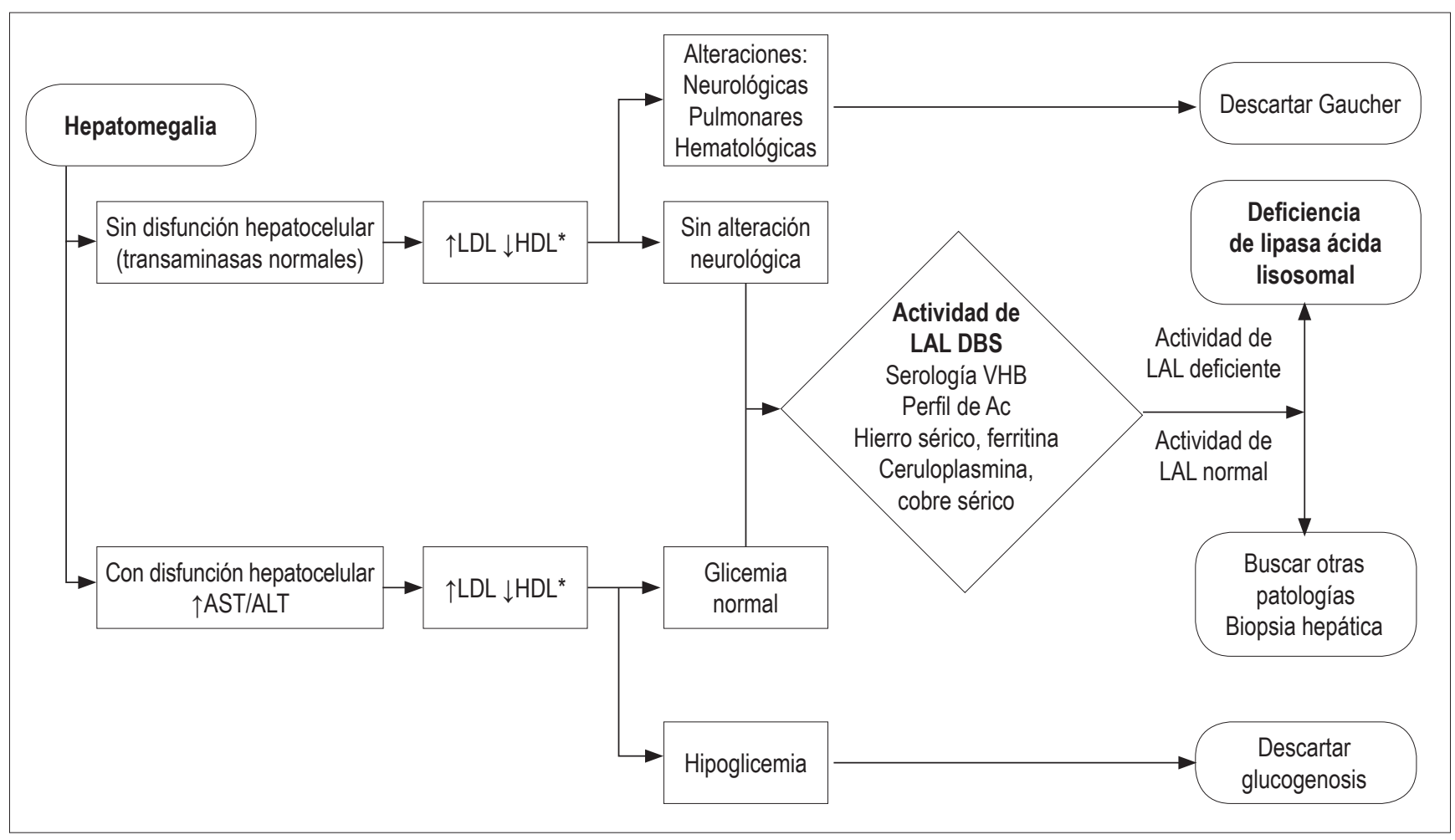

Figura 4. Algoritmo para la sospecha de LAL-D ante el hallazgo de hepatomegalia. *Pediátrica: LDL (mg/dL) $\geq 130$ o HDL (mg/dL) $\leq 45$; adulta: $\mathrm{LDL}(\mathrm{mg} / \mathrm{dL}) \geq 160 \mathrm{o} H \mathrm{HL}(\mathrm{mg} / \mathrm{dL}) \leq 40$ (hombres)/ $\leq 50$ (mujeres). Ac: anticuerpos; Anti-VHC: anticuerpos antivirus de la hepatitis C; VHB: virus de la hepatitis B. 


\section{HEPATOPATÍAS METABÓLICAS}

\section{Enfermedad de Gaucher}

La enfermedad de Gaucher es un desorden autosómico recesivo causado por la deficiencia de glucocerebrosidasa (GBA), una hidrolasa lisosomal. Se estima una incidencia de 1:200 000 .

El diagnóstico de la enfermedad se sospecha cuando a nivel histopatológico (médula ósea, biopsia hepática o bazo) hay visualización de células Gaucher. Sin embargo, la medición de la actividad de GBA en leucocitos o fibroblastos obtenidos de biopsia de piel es el estándar de oro para el diagnóstico (28); adicionalmente, se puede realizar el estudio molecular del gen GBA para su confirmación.

A nivel clínico, esta enfermedad tiene una afectación multisistémica con fenotipos que se dividen en no neuronopática y neuronopática. En los pacientes con enfermedad de Gaucher no neuronopática, las manifestaciones clínicas muestran una gran variabilidad, aunque la mayoría de ellos tienen compromiso hematológico (anemia y trombocitopenia), visceral (hepatoesplenomegalia) y óseo. Además, pueden aparecer complicaciones neurológicas secundarias como consecuencia de compresión nerviosa de la médula espinal debido al colapso vertebral (28-30).

\section{Déficit de esfingomielinasa ácida (EMA) (enfermedad de Niemann-Pick A y B)}

El déficit de EMA es un desorden autosómico recesivo causado por actividad enzimática de la EMA menor al $10 \%$ o por la presencia de mutaciones patogénicas bialélicas en el gen SMPD1 (también llamado gen ASM) (31,32).

Clásicamente, este desorden ha sido divido en neuronopático (enfermedad de Niemann-Pick A) o no neuronopático (enfermedad de Niemann-Pick B) (Tabla 1). Sin embargo, hay formas intermedias entre ambos fenotipos (33).

\section{Glucogenosis}

El glucógeno es un polisacárido formado por moléculas de glucosa unidas entre sí predominantemente por enlaces alfa $1,4(\alpha-1,4)$ y en $7 \%$ a $10 \%$ por enlaces alfa $1,6(\alpha-1,6)$. En el hígado, el glucógeno tiene como misión mantener la glucemia; y en el músculo, se utiliza para la obtención de energía (ATP) durante la contracción muscular $(34,35)$. Se incluyen bajo este término los trastornos genéticos que afectan la vía de la síntesis y degradación del glucógeno y las de su utilización.

Atendiendo a las manifestaciones clínicas, a los criterios de diagnóstico y a su tratamiento, los trastornos genéticos
Tabla 1. Cuadro comparativo entre el síndrome metabólico y LAL-D

\begin{tabular}{|c|c|c|}
\hline & $\begin{array}{l}\text { Síndrome } \\
\text { metabólico }\end{array}$ & LAL-D parcial \\
\hline Peso & Obesidad progresiva & Pérdida de peso \\
\hline Crecimiento & Talla normal-alta & Talla tendencia baja \\
\hline $\begin{array}{l}\text { Aumento de } \\
\text { perímetro } \\
\text { abdominal- } \\
\text { esteatosis }\end{array}$ & $\begin{array}{l}\text { Esteatosis hepática y } \\
\text { grasa visceral }\end{array}$ & $\begin{array}{l}\text { Esteatosis hepática y } \\
\text { esplenomegalia }\end{array}$ \\
\hline C-LDL & $\begin{array}{l}\text { Alto/normal } \\
\text { Mejora con cambios } \\
\text { en estilo de vida/ } \\
\text { medicación }\end{array}$ & $\begin{array}{l}\text { Muy elevado } \\
\text { No mejora con cambios } \\
\text { en estilo de vida/ } \\
\text { medicación }\end{array}$ \\
\hline C-HDL & $\begin{array}{l}\text { Bajo/normal } \\
\text { Aumenta con el } \\
\text { ejercicio }\end{array}$ & $\begin{array}{l}\text { Bajo/normal } \\
\text { No aumenta con el } \\
\text { ejercicio }\end{array}$ \\
\hline Triglicéridos & Alto/normal & Alto/normal \\
\hline Presión arterial & Normal-alta & Normal \\
\hline $\begin{array}{l}\text { Metabolismo de } \\
\text { carbohidratos }\end{array}$ & $\begin{array}{l}\text { Normal/prediabetes/ } \\
\text { DM2 }\end{array}$ & Normal \\
\hline
\end{tabular}

que afectan el metabolismo del glucógeno pueden dividirse en 2 categorías: las que tienen una fisiopatología hepática hipoglucémica y las musculares. Dentro del primer grupo estarían las glucogenosis tipos Ia, Ib, IIIa, IIIb y VI; y dentro del segundo las glucogenosis tipos V, VII y los defectos de la glucólisis que no causan acumulación de glucógeno. Sin embargo, existen entidades que presentan una fisiopatología peculiar, como las glucogenosis tipos II y IV $(34,35)$. La presencia de hiperlipidemias y hepatomegalia en estas glucogenosis obligan a que se incluya dentro de su diagnóstico diferencial LAL-D.

En la Figura 5 se describe el algoritmo diagnóstico para las enfermedades hepáticas metabólicas.

\section{Síndrome metabólico}

Es una constelación de factores de riesgo de origen metabólico interrelacionados, que promueven directamente el desarrollo de enfermedad arteriovascular, DM2, hígado graso y varios cánceres (36).

Según la Federación Internacional de Diabetes, los criterios para este síndrome han sido definidos como la presencia de al menos 3 de los siguientes 5 factores (37):

1. Aumento de la circunferencia abdominal de acuerdo con los criterios del país y de la población específica.

2. Aumento de los triglicéridos $(\geq 150 \mathrm{mg} / \mathrm{dL})$.

3. Disminución del colesterol HDL $(<40 \mathrm{mg} / \mathrm{dL}$ en hombres y $<50 \mathrm{mg} / \mathrm{dL}$ en mujeres). 


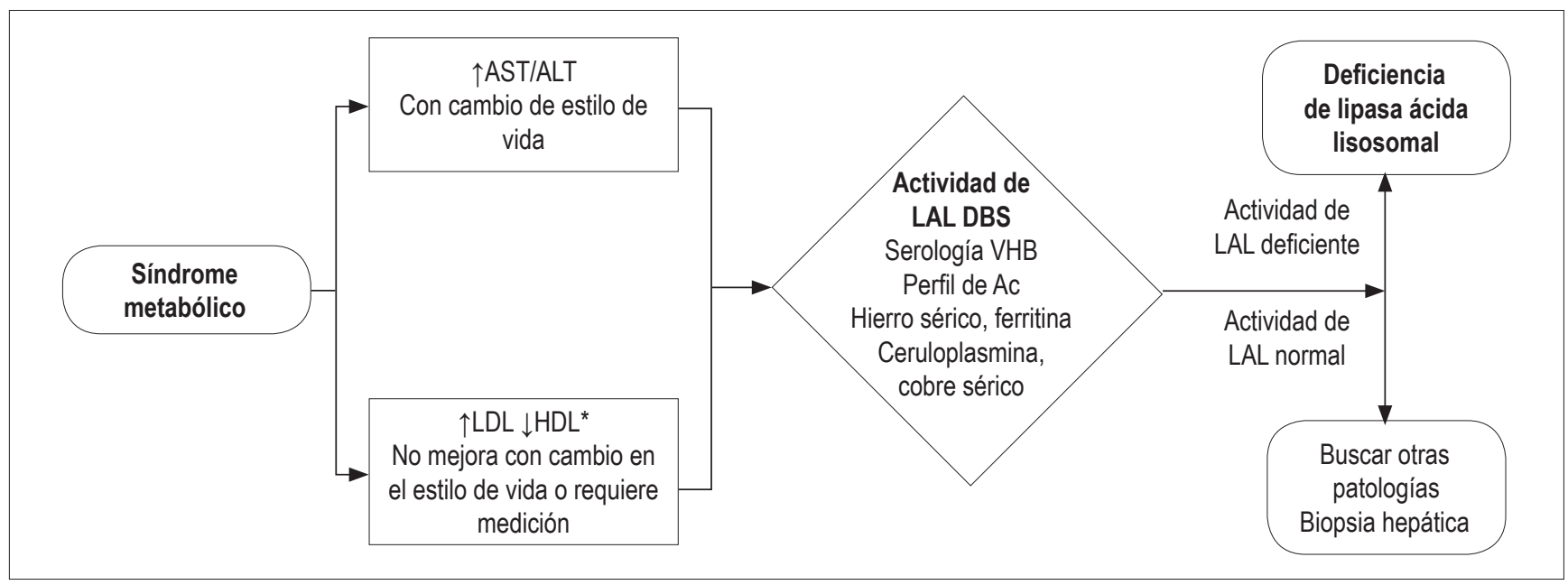

Figura 5. Algoritmo para descartar LAL-D en casos de síndrome metabólico. *Pediátrica: LDL (mg/dL) $\geq 130$ o HDL (mg/dL) $\leq 45$; adulta: LDL $(\mathrm{mg} / \mathrm{dL}) \geq 160$ o HDL $(\mathrm{mg} / \mathrm{dL}) \leq 40$ (hombres) $/ \leq 50$ (mujeres).

4. Aumento de la presión arterial (presión sistólica $\geq 130$ $\mathrm{mm} \mathrm{Hg}$ o presión diastólica $\geq 85 \mathrm{~mm} \mathrm{Hg}$ ).

5. Aumento de la glucosa en ayunas (glicemia $>100 \mathrm{mg} / \mathrm{dL}$ ).

Se han descrito numerosos factores que contribuyen con el desarrollo del síndrome metabólico, incluyendo genéticos, estilo de vida (dieta y actividad física) obesidad y la resistencia a la insulina. Todos estos son elementos importantes en la patogénesis del síndrome metabólico; sin embargo, la resistencia a la insulina es uno de los factores clave que conecta y contribuye para el desarrollo del trastorno. La elevación de los ácidos grasos y los perfiles anormales de adipocitocinas pueden resultar en el establecimiento de una resistencia a la insulina y contribuir con la patogénesis de la enfermedad (38).

En la Tabla 1 se resumen las diferencias entre LAL-D y el síndrome metabólico.

Para la ruta diagnóstica, el consenso de expertos definió que el enfoque del paciente con hallazgos que hacen parte del diagnóstico de síndrome metabólico deben ser las resumidas en la Figura 6.

\section{DISLIPIDEMIAS}

\section{Hipercolesterolemia familiar}

Es una enfermedad de herencia autosómica dominante que lleva a una elevación severa del colesterol total y del LDL. Existen 2 formas de presentación: en homocigosis y en heterocigosis.
Los pacientes homocigotos van a cursar con síntomas consistentes con enfermedad vascular periférica, enfermedad isquémica cardíaca, enfermedad cerebrovascular o estenosis aórtica. Adicionalmente, pueden cursar con síntomas articulares como tendinitis y artralgias, así como la aparición de xantomas cutáneos al nacimiento o en la infancia temprana (39). La mayoría de los pacientes homocigotos sin tratamiento fallecen antes de los 30 años de vida.

Los pacientes heterocigotos generalmente son asintomáticos; sin embargo, pueden cursar con una historia de hipercolesterolemia severa desde la infancia y tardíamente pueden desarrollar xantelasmas o xantomas (39).

Para el diagnóstico de las formas homocigotas y heterocigotas, el hallazgo fundamental son los niveles de colesterol en ausencia de causas secundarias de hipercolesterolemia; pero el diagnóstico definitivo solo podrá hacerse con la confirmación molecular dada por el análisis de los genes LDLR, APOB, o PCSK9 (40).

Los hallazgos en el perfil lipídico son:

- Homocigotos: niveles de colesterol total y LDL >600 $\mathrm{mg} / \mathrm{dL}$ y triglicéridos en el rango de referencia.

- Heterocigotos: niveles de colesterol LDL $>250 \mathrm{mg} / \mathrm{dL}$ en menores de 20 años y mayores de 290-300 mg/dL en adultos. Los triglicéridos están dentro de los rangos de referencia $(41)$.

\section{Hiperlipidemia combinada familiar}

Constituye el trastorno heredado más frecuente del metabolismo lipídico asociado con hiperlipidemia mixta y enfer- 


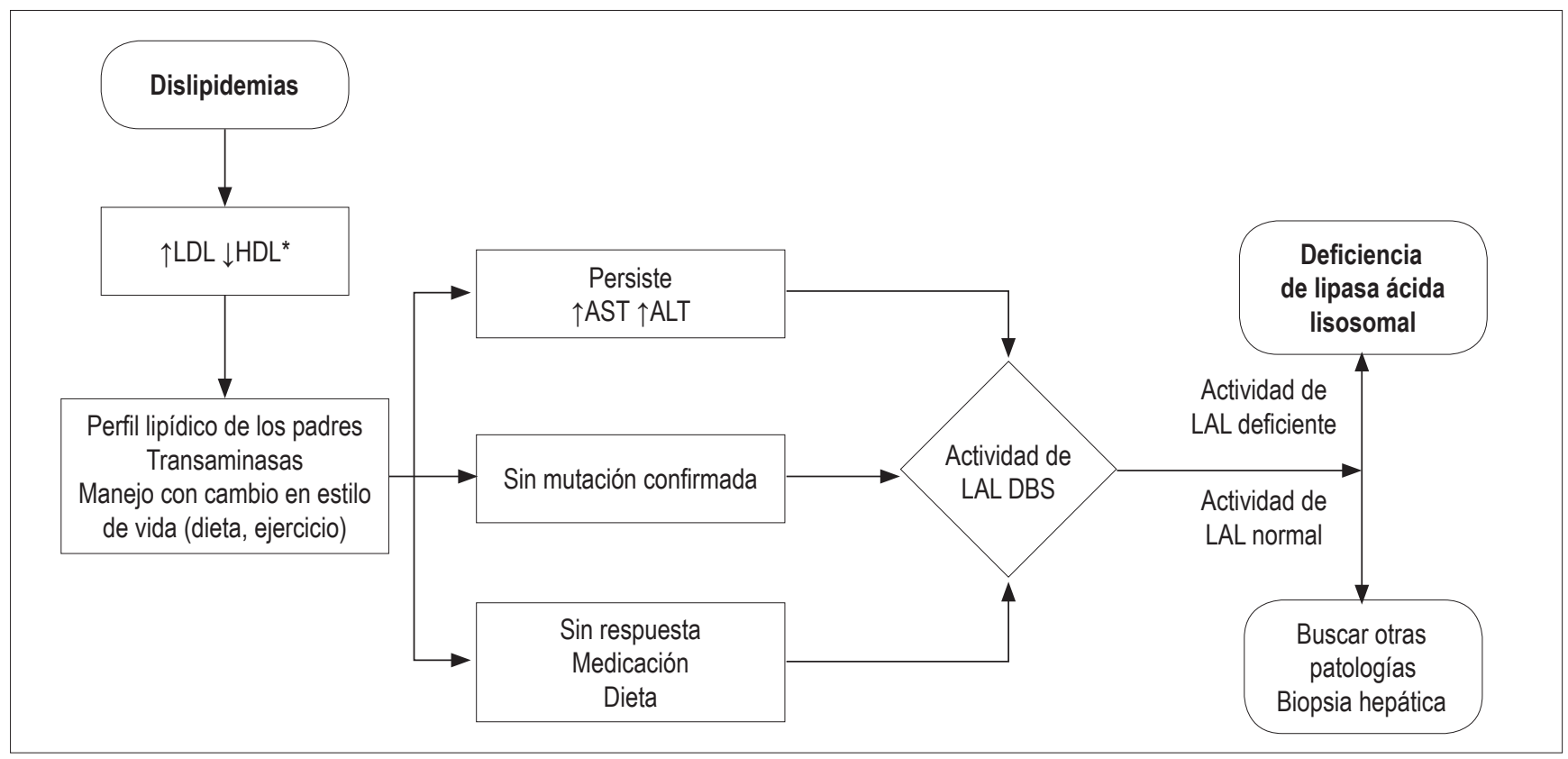

Figura 6. Algoritmo para la sospecha de LAL-D en casos de dislipidemia. ${ }^{*}$ Pediátrica: LDL (mg/dL) $\geq 130$ o HDL (mg/dL) $\leq 45$; adulta: LDL (mg/ $\mathrm{dL}) \geq 160$ o HDL $(\mathrm{mg} / \mathrm{dL}) \leq 40$ (hombres) $/ \leq 50$ (mujeres).

medad cardiovascular prematura. Aunque los síntomas pueden aparecer desde la infancia, usualmente se manifiesta en la segunda década de la vida con hipercolesterolemia o hipertrigliceridemia. Frecuentemente se asocia con numerosas anormalidades metabólicas, como hipertensión arterial, resistencia a la insulina, DM2, obesidad central, esteatosis hepática y síndrome metabólico (42).

Se caracteriza por una herencia autosómica dominante con penetrancia elevada y de etiología oligogénica.

Para el diagnóstico se tienen en cuenta los siguientes criterios:

1. Persona afectada

- En adultos: colesterol total $>240 \mathrm{mg} / \mathrm{dl}$ (o c-LDL $>160 \mathrm{mg} / \mathrm{dL}$ ) y/o triglicéridos $>200 \mathrm{mg} / \mathrm{dL}$.

- En menores de 20 años: colesterol total $>200 \mathrm{mg} /$ dL (o c-LDL > $130 \mathrm{mg} / \mathrm{dL}$ ) o triglicéridos $>120$ $\mathrm{mg} / \mathrm{dL}$.

- Descartar causas secundarias: índice de masa corporal (IMC) $>35 \mathrm{~kg} / \mathrm{m}^{2}$, hemoglobina glucosilada $\left(\mathrm{HbA}_{1 \mathrm{c}}\right)>10 \%$, hipotiroidismo no controlado o etilismo (>40 g alcohol/día).

2. Familia afectada

- 2 o más miembros de primer grado (padres, hermanos o hijos) afectados de hiperlipidemia mixta o de combinaciones de fenotipos, entre hipercolesterolemia pura (IIa), hiperlipidemia mixta (IIb) o hipertrigliceridemia (IV).
- Se excluyen familias con xantomas tendinosos o cifras de c-LDL $>300 \mathrm{mg} / \mathrm{dL}$ en 2 o más familiares de primer grado con fenotipo IIa.

- Historia familiar de enfermedad cardiovascular ateroesclerótica prematura (<60 años) (42).

\section{CONCLUSIÓN}

LAL-D es una patología con una baja prevalencia, posiblemente secundaria al gran subdiagnóstico o diagnóstico errado. Las presentes guías pretenden definir a cuáles pacientes se les debe realizar la prueba que permita hacer el diagnóstico de la enfermedad, con el objeto de que el paciente reciba un tratamiento oportuno y efectivo que permita cambiar su pronóstico y calidad de vida.

Estas guías sirven de referencia para la sospecha diagnóstica en la práctica clínica de médicos generales y especialistas.

\section{REFERENCIAS}

1. Aslanidis C, Ries S, Fehringer P, et al. Genetic and biochemical evidence that CESD and Wolman disease are distinguished by residual lysosomal acid lipase activity. Genomics. 1996;33(1):85-93. Available from: http://www.ncbi.nlm. nih.gov/pubmed/8617513

2. Bernstein DL, Hülkova H, Bialer MG, et al. Cholesteryl ester storage disease: review of the findings in 135 reported patients 
with an underdiagnosed disease.J Hepatol. 2013;58(6):123043. http://dx.doi.org/10.1016/j.jhep.2013.02.014

3. Sloan HR, Fredrickson DS. Enzyme deficiency in cholesteryl ester storage idisease. J Clin Invest. 1972;51(7):1923-6.

4. Abramov A, Schorr S, Wolman M. Generalized xanthomatosis with calcified adrenals. AMA J Dis Child. 1956;91(3):282-6.

5. Jones SA, Valayannopoulos V, Schneider E, et al. Rapid progression and mortality of lysosomal acid lipase deficiency presenting in infants. Genet Med. 2016;18(5):452-8. Available from: http://www.nature.com/doifinder/10.1038/gim.2015.108

6. Fredrickson D. Newly recognized disorders of cholesterol metabolism. Ann Intern Med. 1963;58:718.

7. Fasano T, Pisciotta L, Bocchi L, et al. Lysosomal lipase deficiency: molecular characterization of eleven patients with Wolman or cholesteryl ester storage disease. Mol Genet Metab. 2012;105(3):450-6.

8. Muntoni S, Wiebusch H, Jansen-Rust M, et al. Prevalence of cholesteryl ester storage disease. Arterioscler Thromb Vasc Biol. 2007;27(8):1866-8.

9. Pisciotta L, Fresa R, Bellocchio A, et al. Cholesteryl Ester Storage Disease (CESD) due to novel mutations in the LIPA gene. Mol Genet Metab. 2009;97(2):143-8. http://dx.doi. org/10.1016/j.ymgme.2009.02.007

10. Grabowski G, Charnas L, Du H. Lysosomal acid lipase deficiencies: the Wolman disease/cholesteryl ester storage disease spectrum. Online Metab Mol Bases Inherit Dis. 2012;1-26.

11. Freudenberg F, Bufler P, Ensenauer R, et al. Cholesteryl ester storage disease: an easily missed diagnosis in oligosymptomatic children. Z Gastroenterol. 2013;51(10):1184-7.

12. Scott SA, Liu B, Nazarenko I, et al. Frequency of the cholesteryl ester storage disease common LIPA E8SJM mutation (c.894G >A) in various racial and ethnic groups. Hepatology. 2013;58(3):958-65.

13. Saito S, Ohno K, Suzuki T, et al. Structural bases of Wolman disease and cholesteryl ester storage disease. Mol Genet Metab. 2012;105(2):244-8.

14. Reiner Ž, Guardamagna O, Nair D, et al. Lysosomal acid lipase deficiency--an under-recognized cause of dyslipidaemia and liver dysfunction. Atherosclerosis. 2014;235(1):21-30.

15. Zhang B, Porto AF. Cholesteryl ester storage disease: protean presentations of lysosomal acid lipase deficiency. J Pediatr Gastroenterol Nutr. 2013;56(6):682-5. Available from: http://content.wkhealth.com/linkback/openurl?sid=WKP TLP:landingpage\&an $=00005176-201306000-00020$

16. Nchimi A, Rausin L, Khamis J. Ultrasound appearance of bowel wall in Wolman's disease. Pediatr Radiol. 2003;33(4):284-5.

17. Jones SA, Bernstein D, Bialer M, et al. Severe and rapid disease course in the natural history of infants with lysosomal acid lipase deficiency. Mol Genet Metab. 2014;111(2):S578. Available from: http://www.sciencedirect.com/science/ article/pii/S109671921300557X\%5Cnhttp://linkinghub. elsevier.com/retrieve/pii/S109671921300557X
18. Elleder M, Chlumská A, Hyánek J, et al. Subclinical course of cholesteryl ester storage disease in an adult with hypercholesterolemia, accelerated atherosclerosis, and liver cancer. J Hepatol. 2000;32(3):528-34.

19. Elleder M, Ledvinová J, Cieslar P, et al. Subclinical course of cholesterol ester storage disease (CESD) diagnosed in adulthood. Report on two cases with remarks on the nature of the liver storage process. Virchows Arch A Pathol Anat Histopathol. 1990;416(4):357-65.

20. Ameis D, Brockmann G, Knoblich R, et al. A 5 ' splice-region mutation and a dinucleotide deletion in the lysosomal acid lipase gene in two patients with cholesteryl ester storage disease. J Lipid Res. 1995;36(2):241-50.

21. Riva S, Spada M, Sciveres M, et al. Hepatocarcinoma in a child with cholesterol ester storage disease. Dig Liver Dis. 2008;40(9):784.

22. Drebber U, Andersen M, Kasper HU, et al. Severe chronic diarrhea and weight loss in cholesteryl ester storage disease: a case report. World J Gastroenterol. 2005;11(15):2364-6.

23. Hamilton J, Jones I, Srivastava R, et al. A new method for the measurement of lysosomal acid lipase in dried blood spots using the inhibitor Lalistat 2. Clin Chim Acta. 2012;413(1516):1207-10. http://dx.doi.org/10.1016/j.cca.2012.03.019

24. Civallero G, Michelin K, de Mari J, et al. Twelve different enzyme assays on dried-blood filter paper samples for detection of patients with selected inherited lysosomal storage diseases. Clin Chim Acta. 2006;372(1-2):98-102.

25. Hůlková $H$, Elleder $M$. Distinctive histopathological features that support a diagnosis of cholesterol ester storage disease in liver biopsy specimens. Histopathology. 2012;60(7):1107-13.

26. Chalasani N, Younossi Z, Lavine JE, et al. The diagnosis and management of non-alcoholic fatty liver disease: practice guideline by the American Gastroenterological Association, American Association for the Study of Liver Diseases, and American College of Gastroenterology. Gastroenterology. 2012;142(7):1592-609. http://dx.doi.org/10.1053/j.gastro.2012.04.001

27. Wolf $\mathrm{AD}$, Lavine JE. Hepatomegaly in neonates and children. Pediatr Rev. 2000;21(9):303-10.

28. Linari S, Castaman G. Clinical manifestations and management of Gaucher disease. Clin Cases Miner Bone Metab. 2015;12(2):157-64. Available from: http://www.pubmedcentral.nih.gov $/$ articlerender.fcgi $?$ artid $=4625773 \&$ tool $=p$ mcentrez\&rendertype $=$ abstract

29. vom Dahl S, Harzer K, Rolfs A, et al. Hepatosplenomegalic lipidosis: what unless Gaucher? Adult cholesteryl ester storage disease (CESD) with anemia, mesenteric lipodystrophy, increased plasma chitotriosidase activity and a homozygous lysosomal acid lipase - 1 exon 8 splice junction mutation. J Hepatol. 1999;31(4):741-6.

30. Masi L, Brandi ML. Gaucher disease: the role of the specialist on metabolic bone diseases. Clin Cases Miner Bone Metab. 2015;12(2):165-9. 
31. Zampieri S, Filocamo M, Pianta A, et al. SMPD1 Mutation Update: Database and Comprehensive Analysis of Published and Novel Variants. Hum Mutat. 2016;37(2):139-47.

32. Rhein C, Mühle C, Kornhuber J, et al. Alleged Detrimental Mutations in the SMPD1 Gene in Patients with NiemannPick Disease. Int J Mol Sci. 2015;16(6):13649-52. Available from: http://www.pubmedcentral.nih.gov/articlerender.fcgi ?artid=4490514\&tool=pmcentrez\&rendertype $=$ abstract

33. McGovern MM, Wasserstein MP, Giugliani R, et al. A prospective, cross-sectional survey study of the natural history of Niemann-Pick disease type B. Pediatrics. 2008;122(2):e341-9.

34. Bodamer OA, Feillet F, Lane RE, et al. Utilization of cornstarch in glycogen storage disease type Ia. Eur J Gastroenterol Hepatol. 2002;14(11):1251-6. Available from: http://eutils.ncbi.nlm.nih.gov/entrez/eutils/elink. fcgi?dbfrom $=$ pubmed\&id $=12439121 \&$ retmode $=$ ref $\& \mathrm{~cm}$ $\mathrm{d}=$ prlinks\%5Cnpapers3://publication/uuid/CB712C8ED63E-4AC0-9B42-4C5DF0A2230C

35. Avaria, M, Kleinsteuber S. Trastorno del metabolismo de hidratos de carbono. En: Colombo M, Cornejo Espinoza V, Raimann Ballas EA (editoras). Errores innatos en el metabolismo del niño. 3. a edición. Santiago: Editorial Universitaria; 2010. p. 458-66.

36. Grundy SM, Brewer HB Jr, Cleeman JI, et al. Definition of metabolic syndrome: Report of the National Heart, Lung, and Blood Institute/American Heart Association conference on scientific issues related to definition. Circulation. 2004;109(3):433-8.

37. Alberti KGMM, Eckel RH, Grundy SM, et al. Harmonizing the metabolic syndrome: a joint interim statement of the International Diabetes Federation Task Force on Epidemiology and Prevention; National Heart, Lung, and Blood Institute; American Heart Association; World Heart Federation; International Atherosclerosis Society; and International Association for the Study of Obesity. Circulation. 2009;120(16):1640-5. Available from: http://circ.ahajournals.org/content/120/16/1640. abstract \% 5 Cnhttp: / / circ.ahajournals.org/content/120/16/1640.full.pdf

38. Weiss R, Bremer AA, Lustig RH. What is metabolic syndrome, and why are children getting it? Ann N Y Acad Sci. 2013;1281:123-40.

39. Varghese MJ. Familial hypercholesterolemia: A review. Ann Pediatr Cardiol. 2014;7(2):107-17. Available from: http:// www.annalspc.com/text.asp?2014/7/2/107/132478

40. Wiegman A, Gidding SS, Watts GF, et al. Familial hypercholesterolaemia in children and adolescents: gaining decades of life by optimizing detection and treatment. Eur Heart J. 2015;36(36):2425-37.

41. Nordestgaard BG, Chapman MJ, Humphries SE, et al. Familial hypercholesterolaemia is underdiagnosed and undertreated in the general population: guidance for clinicians to prevent coronary heart disease: consensus statement of the European Atherosclerosis Society. Eur Heart J. 2013;34(45):3478-90a.

42. Mata P, Alonso R, Ruíz-García A, et al. Hiperlipidemia familiar combinada: documento de consenso. Aten Primaria. 2014;46(8):440-6. http://dx.doi.org/10.1016/j. aprim.2014.04.013 